\title{
Algebraic genericity of strict-order integrability
}

\author{
by \\ Luis BERNAL-GonzÁLEZ (Sevilla)
}

\begin{abstract}
We provide sharp conditions on a measure $\mu$ defined on a measurable space $X$ guaranteeing that the family of functions in the Lebesgue space $L^{p}(\mu, X)(p \geq 1)$ which are not $q$-integrable for any $q>p$ (or any $q<p$ ) contains large subspaces of $L^{p}(\mu, X)$ (without zero). This improves recent results due to Aron, García, Muñoz, Palmberg, Pérez, Puglisi and Seoane. It is also shown that many non- $q$-integrable functions can even be obtained on any nonempty open subset of $X$, assuming that $X$ is a topological space and $\mu$ is a Borel measure on $X$ with appropriate properties.
\end{abstract}

1. Introduction and aim of this paper. The study of linear properties of sets of mathematical objects with a priori no linear structure has recently attracted the attention of a growing number of mathematicians. This paper intends to shed some light on this topic, in the special framework of spaces of integrable functions.

In this respect, let us recall some recent terminology introduced in [1], [3], [5] and [8]. Assume that $E$ is a topological vector space. Then a subset $A$ of $E$ is called

- lineable if $A \cup\{0\}$ contains an infinite-dimensional vector subspace,

- dense-lineable or algebraically generic whenever $A \cup\{0\}$ contains a dense vector subspace of $X$,

- spaceable if $A \cup\{0\}$ contains some infinite-dimensional closed vector subspace.

It is clear that dense-lineability implies lineability if $E$ is infinite-dimensional.

If $\mu$ is a cardinal number, then a subset $A$ of $E$ is said to be $\mu$-lineable if $A \cup\{0\}$ contains a vector subspace of dimension $\mu$. Note that if $E$ is an infinite-dimensional separable Baire topological vector space, then $\operatorname{dim}(E)=c$, the cardinality of the continuum. Hence $c$ is the maximal dimension of any vector subspace of $E$.

2010 Mathematics Subject Classification: Primary 28A25; Secondary 28C15, 46E30.

Key words and phrases: dense-lineability, maximal lineability, Lebesgue space, strict order of integration, Borel measure. 
If the last notion is combined with algebraic genericity, the following concept arises naturally.

Definition 1.1. Let $E$ be a topological vector space. Then we say that a subset $A \subset E$ is maximal dense-lineable if $A \cup\{0\}$ contains a dense vector subspace $M$ such that $\operatorname{dim}(M)=\operatorname{dim}(E)$.

Note that it is not clear whether maximal lineability (i.e., $\operatorname{dim}(E)$-lineability) plus dense-lineability implies maximal dense-lineability.

For each $p \in[1, \infty)$ and each interval $I \subset \mathbb{R}$, let us consider the Lebesgue space $L^{p}(I)$ of (classes of) measurable functions $f: I \rightarrow \mathbb{R}$ such that $|f|^{p}$ is integrable on $I$ with respect to the Lebesgue measure. We also consider the space $\ell_{p}$ of real sequences $\left(a_{n}\right)$ that are $p$-summable, that is, $\sum_{n=1}^{\infty}\left|a_{n}\right|^{p}$ $<\infty$. In 2008, Muñoz, Palmberg, Puglisi and Seoane [9] proved the following assertions:

- If $I$ is a bounded interval and $q>p \geq 1$ then $L^{p}(I) \backslash L^{q}(I)$ is lineable.

- If $J$ is an unbounded interval and $p>q \geq 1$ then $L^{p}(J) \backslash L^{q}(J)$ is lineable.

- If $p>q \geq 1$ then $\ell_{p} \backslash \ell_{q}$ is lineable.

More precisely, it is proved in [9] that these sets are c-lineable. Aron, García, Pérez and Seoane 2] have recently completed these results by showing that, under the same conditions, the three sets mentioned above are dense-lineable respectively in $L^{p}(I), L^{p}(J)$ and $\ell_{p}$. Note that $L^{q}(I) \subset L^{p}(I)$ if $q>p$, and $\ell_{q} \subset \ell_{p}$ if $p>q$, but none of the spaces $L^{p}(J), L^{q}(J)$ is included in the other if $p \neq q$.

Our aim in this paper is to unify, extend and improve both of the above mentioned results of [2] and [9], according to the following points:

- We obtain vector subspaces exhibiting simultaneously density and maximal dimension.

- The intervals $I, J$ endowed with the Lebesgue measure are replaced by rather general measure spaces, so we cover the case of $\ell_{p}$ too. The conditions given on the measures will be sharp.

- The parameter $q$ runs over all real numbers $>p$ (or $<p$ ), that is, $q$ is not fixed.

- In the case of Borel measures, many non- $q$-integrable functions are obtained even on every nonempty open subset.

The precise statements will be given in Sections 3 and 4 . Section 2 is devoted to the necessary background. In Section 5 we make some remarks and propose a number of problems.

2. Preliminaries. Let $(X, \mathcal{M}, \mu)$ be a measure space, with $\mu$ a positive measure, and let $p \in[1, \infty)$. As usual, $L^{p}(\mu, X)$ will denote the vector space 
of all (Lebesgue classes of) measurable functions $f: X \rightarrow \mathbb{R}$ such that $|f|^{p}$ is integrable on $X$. It becomes a Banach space under the norm $\|f\|_{p}=$ $\left(\int_{X}|f|^{p} d \mu\right)^{1 / p}$. If $p=\infty, L^{\infty}(\mu, X)$ represents the space of all Lebesgue classes of essentially bounded measurable functions $f: X \rightarrow \mathbb{R}$. It becomes a Banach space under the norm $\|f\|=\inf \{M>0:|f| \leq M \mu$-almost everywhere in $X\}$.

We assume that the reader is familiar with some standard topological terminology and with a number of related properties, which can be found in any reasonable book on general topology. Anyway, we recall that a topological space $X$ is said to be: perfect if it lacks isolated points; regular at a point $x_{0} \in X$ if, given a closed set $F$ with $x_{0} \notin F$, there are open sets $A, B$ such that $x_{0} \in A, F \subset B$ and $A \cap B=\emptyset$; first-countable at a point $x_{0} \in X$ if $x_{0}$ possesses a countable fundamental system of neighborhoods.

Now, we recall a number of special types of measures defined either on general $\sigma$-algebras or on the $\sigma$-algebra of Borel sets of a topological space. Let $(X, \mathcal{M}, \mu)$ be a measure space. We say that $\mu$ is semifinite if $\mu(A)=\sup \{\mu(B): B \in \mathcal{M}, B \subset A$ and $\mu(B)<\infty\}$ for each set $A \in \mathcal{M}$, while $\mu$ is called nonatomic if there is no atom in $\mathcal{M}$. Recall that a set $A \in \mathcal{M}$ is said to be an atom for $\mu$ if $\mu(A)>0$ and if, for every $B \in \mathcal{M}$ with $B \subsetneq A$, one has $\mu(B)=0$ and $\mu(A \backslash B)=0$.

Assume that $X$ is a topological space and that $\mu$ is a Borel measure on $X$, that is, $\mu$ is a positive measure defined on a $\sigma$-algebra $\mathcal{M}$ containing the Borel sets of $X$. Then we say that $\mu$ has full support whenever $\mu(U)>0$ for every nonempty open set $U$, and $\mu$ is continuous if $\mu(\{x\})=0$ for all $x \in X$. Finally, $\mu$ is called regular if $X$ is a Hausdorff locally compact space and the following three properties hold:

(a) $\mu(C)<\infty$ for every compact set $C \subset X$,

(b) $\mu(A)=\inf \{\mu(U): U$ is open and $A \subset U\}$ for all $A \in \mathcal{M}$,

(c) $\mu(U)=\sup \{\mu(C): C$ is compact in $X$ and $C \subset U\}$ for each open set $U \subset X$.

For instance, the Lebesgue measure on any interval of $\mathbb{R}$ is continuous, regular and has full support. It is known that each $\sigma$-finite measure is semifinite, and that every regular measure $\mu$ also satisfies (c) above if one replaces $U$ by any set $\sigma$-finite set $A \in \mathcal{M}$ (see [10, Proposition 22.5]).

In Sections 3 and 4 the following two lemmas will be respectively needed. Lemma 2.1 is a strengthening of Theorem 2.1 in [6]. The nuance is that this time the dimension of the resulting vector subspace is specified. Lemma 2.2 has a narrow scope and replaces a topological vector space by a topological group.

Lemma 2.1. Assume that $E$ is a metrizable separable topological vector space. Suppose that $\Gamma$ is a family of linear subspaces of $E$ such that $\bigcap_{S \in \Gamma} S$ 
is dense in $E$ and $\bigcap_{S \in \Gamma}(E \backslash S) \cup\{0\}$ contains a vector space of dimension $\mu$, where $\mu$ is an infinite cardinal number. Then $\bigcap_{S \in \Gamma}(E \backslash S) \cup\{0\}$ contains a dense vector subspace of dimension $\mu$.

Proof. From the hypothesis, we can choose a dense countable set $\left\{z_{n}\right\}_{n \geq 1}$ in $E$ as well as a translation-invariant distance $d$ defining the topology of $E$. By denseness, we can also take, for each $n \in \mathbb{N}:=\{1,2, \ldots\}$, a vector $y_{n} \in \bigcap_{S \in \Gamma} S$ such that

$$
d\left(y_{n}, z_{n}\right)<1 / n
$$

By hypothesis, there exists a linearly independent family $\left\{v_{\alpha}\right\}_{\alpha \in J} \subset E$ such that $\operatorname{card}(J)=\mu$ and

$$
L \backslash\{0\} \subset \bigcap_{S \in \Gamma}(E \backslash S),
$$

where we have set $L:=\operatorname{span}\left(\left\{v_{\alpha}: \alpha \in J\right\}\right)$. Since $\mu$ is infinite, we can split $J$ into infinitely many mutually disjoint nonempty sets, say $J=\bigcup_{n=1}^{\infty} J_{n}$. Now, as scalar multiplication is continuous on $E$, there exists a set $\left\{\varepsilon_{\alpha}\right\}_{\alpha \in J} \subset$ $(0, \infty)$ such that

$$
d\left(\varepsilon_{\alpha} v_{\alpha}, 0\right)<1 / n \quad\left(\alpha \in J_{n}, n \in \mathbb{N}\right) .
$$

Next, we define $x_{n, \alpha}:=y_{n}+\varepsilon_{\alpha} v_{\alpha}\left(\alpha \in J_{n}, n \in \mathbb{N}\right)$ and

$$
D:=\operatorname{span}\left(\left\{x_{n, \alpha}: \alpha \in J_{n}, n \in \mathbb{N}\right\}\right),
$$

the linear span of the vectors $x_{n, \alpha}$.

For each $n \in \mathbb{N}$, choose $\alpha_{n} \in J_{n}$ and consider the vector $u_{n}:=x_{n, \alpha_{n}}$. By (1), (2), the triangle inequality and the translation-invariance of $d$,

$$
\begin{aligned}
d\left(u_{n}, z_{n}\right) & \leq d\left(y_{n}+\varepsilon_{\alpha_{n}} v_{\alpha_{n}}, y_{n}\right)+d\left(y_{n}, z_{n}\right) \\
& =d\left(\varepsilon_{\alpha_{n}} v_{\alpha_{n}}, 0\right)+d\left(y_{n}, z_{n}\right)<2 / n \rightarrow 0 \quad(n \rightarrow \infty) .
\end{aligned}
$$

Hence $d\left(u_{n}, z_{n}\right) \rightarrow 0$. Since $\left\{z_{n}\right\}_{n \geq 1}$ is dense and $E$ is perfect (because $E$ is a topological vector space), we derive that $\left\{u_{n}\right\}_{n \geq 1}$ is dense. Consequently, $D$ is a dense linear subspace of $E$.

Let us prove that $D \backslash\{0\} \subset \bigcap_{S \in \Gamma}(E \backslash S)$. To this end, fix $x \in D \backslash\{0\}$. Then there exist $N \in \mathbb{N}$, scalars $c_{1}, \ldots, c_{N}$ with $c_{N} \neq 0$ and indices $\beta_{j} \in J_{j}$ $(j=1, \ldots, N)$ satisfying $x=c_{1} x_{1, \beta_{1}}+\cdots+c_{N} x_{N, \beta_{N}}$, that is,

$$
x=c_{1} y_{1}+\cdots+c_{N} y_{N}+c_{1} \varepsilon_{\beta_{1}} v_{\beta_{1}}+\cdots+c_{N} \varepsilon_{\beta_{N}} v_{\beta_{N}} .
$$

Assume, towards a contradiction, that $x \notin \bigcap_{S \in \Gamma}(E \backslash S)$. Then there would be some $S_{0} \in \Gamma$ for which $x \in S_{0}$. But $y_{1}, \ldots, y_{N} \in \bigcap_{S \in \Gamma} S \subset S_{0}$ and $S_{0}$ is a linear subspace, so

$$
x-\left(c_{1} y_{1}+\cdots+c_{N} y_{N}\right) \in S_{0} .
$$


Since $c_{N} \varepsilon_{\beta_{N}} \neq 0$ and the vectors $v_{\beta_{j}}$ are linearly independent, we deduce that

$$
c_{1} \varepsilon_{\beta_{1}} v_{\beta_{1}}+\cdots+c_{N} \varepsilon_{\beta_{N}} v_{\beta_{N}} \in L \backslash\{0\} \subset \bigcap_{S \in \Gamma}(E \backslash S),
$$

which contradicts (4) because of (3).

Finally, we have to demonstrate that $\operatorname{dim}(D)=\mu$. Since $\operatorname{card}\{(n, \alpha)$ : $\left.\left.\alpha \in J_{n}, n \in \mathbb{N}\right\}\right)=\operatorname{card}\left(\bigcup_{n=1}^{\infty} J_{n}\right)=\mu$, it is enough to show that the family $\left\{x_{n, \alpha}\right\}_{\alpha \in J_{n}, n \in \mathbb{N}}$ is linearly independent. This is easy: assume that the right hand side of (3) is 0 . Fix any $S_{0} \in \Gamma$. Then $c_{1} y_{1}+\cdots+c_{N} y_{N} \in S_{0}$. Therefore

$$
-\left(c_{1} \varepsilon_{\beta_{1}} v_{\beta_{1}}+\cdots+c_{N} \varepsilon_{\beta_{N}} v_{\beta_{N}}\right) \in S_{0} \cap\left[\bigcap_{S \in \Gamma}(E \backslash S) \cup\{0\}\right]=\{0\} .
$$

Thus $c_{1} \varepsilon_{\beta_{1}} v_{\beta_{1}}+\cdots+c_{N} \varepsilon_{\beta_{N}} v_{\beta_{N}}=0$. But the vectors $v_{\alpha}$ are linearly independent, so $c_{j} \varepsilon_{\beta_{j}}=0$ for all $j$, and consequently $c_{1}=\cdots=c_{N}=0$.

Lemma 2.2. Assume that $(E, *)$ is a topological group. Suppose that $\Gamma$ is a family of subgroups of $E$ such that $\bigcap_{S \in \Gamma} S$ is dense in $E$. Then the set $\bigcap_{S \in \Gamma}(E \backslash S)$ is either empty or dense in $E$.

Proof. Suppose that $\bigcap_{S \in \Gamma}(E \backslash S) \neq \emptyset$, so that it has at least one element, say $x_{0}$. Since the "translation" $x \in E \mapsto x * x_{0} \in E$ is a homeomorphism from $E$ onto itself, the set $A:=\left\{x * x_{0}: x \in \bigcap_{S \in \Gamma} S\right\}$ is dense in $E$. Therefore, it is enough to show that $A \subset \bigcap_{S \in \Gamma}(E \backslash S)$. To see this, assume towards a contradiction that there is an element $y=x * x_{0} \in A \backslash \bigcap_{S \in \Gamma}(E \backslash S)$, where $x \in \bigcap_{S \in \Gamma} S$. Then there exists $S_{0} \in \Gamma$ such that $x * x_{0} \in S_{0}$. Hence $x_{0}=x^{-1} *\left(x * x_{0}\right)$ also belongs to $S_{0}$ because $S_{0}$ is a subgroup. This is the desired contradiction.

Next, we state two propositions that are surely well known, but since we have not been able to find a reference, the proofs will be furnished. We first isolate a property to be used later. A measure space $(X, \mathcal{M}, \mu)$ is said to have property $(\sigma)$ if there is a countable set $\mathcal{S} \subset \mathcal{M}$ satisfying the following: given $M \in \mathcal{M}$ with $\mu(M)<\infty$ and $\varepsilon>0$, there exists $A \in \mathcal{S}$ such that $\mu(M \triangle A)<\varepsilon$. By $A \Delta B$ we have denoted the symmetric difference $(A \backslash B) \cup(B \backslash A)$. As usual, $\mathbb{Q}$ will stand for the set of rational numbers, and $\chi_{A}$ for the indicator function of a subset $A$ of $X$.

Proposition 2.3. Let $p \in[1, \infty)$ and $(X, \mathcal{M}, \mu)$ be a measure space.

(a) If $(\sigma)$ is satisfied, then $L^{p}(\mu, X)$ is separable.

(b) If $X$ is a Hausdorff second-countable locally compact topological space and $\mu$ is a regular measure on $X$, then $L^{p}(\mu, X)$ is separable.

Proof. According to [12, Chapter 3], the set $S_{t}$ of step functions, i.e., of measurable functions $f: X \rightarrow \mathbb{R}$ such that $f(X)$ is finite and $\mu(\{x \in X$ : $f(x) \neq 0\})<\infty$, is dense in $L^{p}(\mu, X)$ for any $p \in[1, \infty)$ and any positive 
measure on an arbitrary measurable space $X$. Now, assume that $\mu$ is as in (a), and consider the countable set $\mathcal{S} \subset \mathcal{M}$ furnished by $(\sigma)$. Since the set $\mathcal{A}:=\left\{\sum_{i=1}^{N} q_{i} \chi_{A_{i}}: q_{i} \in \mathbb{Q}, A_{i} \in \mathcal{S}\right.$ for $\left.i \in\{1, \ldots, N\}, N \in \mathbb{N}\right\}$ is countable and $S_{t}$ is dense in $L^{p}(\mu, X)$, it is enough to prove that, given $\varepsilon>0$ and $f \in S_{t}$, there exists $g \in \mathcal{A}$ such that $\|f-g\|_{p}<\varepsilon$. This is easy: just take into account the denseness of $\mathbb{Q}$ in $\mathbb{R}$, the identities $\chi_{A}=\chi_{A}^{p}$, $\left|\chi_{A}-\chi_{B}\right|=\chi_{A \triangle B}$ and the elementary inequality $\left(\sum_{i=1}^{N} a_{i}\right)^{p} \leq N^{p}\left(\sum_{i=1}^{N} a_{i}^{p}\right)$ for $a_{i} \in[0, \infty)$. This demonstrates (a).

Now, assume that $X$ and $\mu$ are as in (b). Select a countable open basis $\mathcal{U}$ for $X$ and define $\mathcal{S}$ to be the family of all finite unions of members of $\mathcal{U}$. Then $\mathcal{S}$ is a countable subfamily of $\mathcal{M}$. Therefore, it is sufficient to prove that $(\sigma)$ is satisfied with $\mathcal{S}$. For this, fix $\varepsilon>0$ and $M \in \mathcal{M}$ with $\mu(M)<\infty$. Since $\mu$ is regular, we can find a compact set $K$ and an open set $U$ satisfying

$$
K \subset M \subset U \text { and } \mu(U)-\varepsilon / 2<\mu(M)<\mu(K)+\varepsilon / 2 .
$$

Fix $x \in K$. Then $x \in U$, so there is $B_{x} \in \mathcal{U}$ with $x \in B_{x} \subset U$, because $\mathcal{U}$ is an open basis. Therefore the family $\left\{B_{x}: x \in K\right\}$ is an open covering of $K$. From the compactness of $K$, there exists a finite subfamily $\left\{B_{x_{j}}: j=\right.$ $1, \ldots, J\}$ such that $K \subset \bigcup_{j=1}^{J} B_{x_{j}}=: A$. Then $A \in \mathcal{S}$ and $K \subset A \subset U$. From this and (5), one derives that $M \triangle A \subset U \backslash K$ and $\mu(M \triangle A) \leq$ $\mu(U \backslash K)=\mu(U)-\mu(K)<\varepsilon$. Consequently, $\mathcal{S}$ witnesses property $(\sigma)$, and by (a) the proof is finished.

The last proposition can be applied, of course, to the spaces $L^{p}(I)$ and $\ell_{p}$, where $p \in[0, \infty)$ and $I$ is any interval of $\mathbb{R}$. Indeed, the Lebesgue measure and the counting measure on $\mathbb{N}$ are special instances of (b). It is well known that, on the contrary, $L^{\infty}(\mu, X)$ is seldom separable.

Note that the sufficient condition for nonseparability furnished in the proposition below is rather common, and works, again, for the Lebesgue measure as well as the counting measure on $\mathbb{N}$.

Proposition 2.4. Let $(X, \mathcal{M}, \mu)$ be a measure space such that there is an infinite family $\mathcal{N} \subset \mathcal{M}$ whose members are pairwise disjoint and $0<$ $\mu(A)<\infty$ for all $A \in \mathcal{N}$. Then $L^{\infty}(\mu, X)$ is not separable.

Proof. Select a sequence $\left\{A_{n}\right\}_{n \geq 1}$ of mutually disjoint sets in $\mathcal{N}$ with positive measure. Consider the family $\mathcal{F}:=\left\{B_{S}=\bigcup_{n \in S} A_{n}: S \subset \mathbb{N}\right\}$, and let $B_{S}, B_{\tilde{S}}$ be distinct members of $\mathcal{F}$. Then $S \Delta \tilde{S} \neq \emptyset$ and $\left|\chi_{B_{S}}-\chi_{B_{\tilde{S}}}\right|$ $=\chi_{B_{S} \Delta B_{\tilde{S}}}$. Hence $\left|\chi_{B_{S}}-\chi_{B_{\tilde{S}}}\right|=1$ on the set $B_{S} \triangle B_{\tilde{S}}$, which has positive measure. Consequently, the open norm-balls $B\left(\chi_{F}, 1 / 2\right)(F \in \mathcal{F})$ are mutually disjoint. The nonseparability of our space follows from the fact that $\mathcal{F}$ is uncountable. 
We finish this section with a purely topological preliminary result, to be used in Section 3. If $A \subset X$, where $X$ is a topological space, then $\bar{A}$ will stand for the closure of $A$.

Lemma 2.5. Let $X$ be a $T_{1}$ topological space. Assume that $x_{0}$ is a nonisolated point of $X$ such that $X$ is regular and first-countable at $x_{0}$. Then, for each open neighborhood $U$ of $x_{0}$, there exists a fundamental system $\left\{U_{n}\right\}_{n \geq 1}$ of open neighborhoods of $x_{0}$ such that

$$
\overline{U_{n+1}} \subset U_{n} \subset U \text { and } \quad U_{n} \backslash \overline{U_{n+1}} \neq \emptyset \quad \text { for all } n \in \mathbb{N} .
$$

Proof. Fix an open neighborhood $U$ of $x_{0}$. Since $X$ is first-countable at $x_{0}$, there exists a fundamental system $\left\{V_{n}\right\}_{n \geq 1}$ of open neighborhoods of $x_{0}$. By replacing, if necessary, each $V_{n}$ by $U \cap V_{1} \cap \cdots \cap V_{n}$, we can assume that $V_{n+1} \subset V_{n} \subset U$ for every $n \in \mathbb{N}$. Pick $x_{1} \in V_{1} \backslash\left\{x_{0}\right\}$, which is possible because $x_{0}$ is not isolated. Now, regularity at $x_{0}$ yields open sets $A_{1}, B_{1}$ satisfying $x_{0} \in A_{1},\left(X \backslash V_{1}\right) \cup\left\{x_{1}\right\} \subset B_{1}$ and $A_{1} \cap B_{1}=\emptyset$ (note that $\left\{x_{1}\right\}$ is closed because $X$ is $T_{1}$ ). Therefore $V_{1} \backslash\left\{x_{1}\right\} \supset X \backslash B_{1} \supset A_{1}$. But $X \backslash B_{1}$ is closed, whence $V_{1} \backslash\left\{x_{1}\right\} \supset \overline{A_{1}}$. Define $U_{1}:=V_{1}$ and $U_{2}:=V_{2} \cap A_{1}$. On the one hand, $U_{1}, U_{2}$ are open sets containing $x_{0}$ with $U_{1} \subset V_{1}, U_{2} \subset V_{2}$. On the other hand, $\overline{U_{2}} \subset \overline{A_{1}} \subset V_{1}=U_{1}$ and $x_{1} \in U_{1} \backslash \overline{A_{1}} \subset U_{1} \backslash \overline{U_{2}}$. Hence $U_{1} \backslash \overline{U_{2}} \neq \emptyset$. By starting with $U_{2}$, we can pick $x_{2} \in U_{2} \backslash\left\{x_{0}\right\}$, and a similar process yields an open set $U_{3}$ satisfying $x_{0} \in U_{3}, \overline{U_{3}} \subset U_{2}$, $U_{3} \subset V_{3}$ and $x_{2} \in U_{2} \backslash \overline{U_{3}}$ (so $U_{2} \backslash \overline{U_{3}} \neq \emptyset$ ). It is plain that this procedure generates the desired sequence $\left\{U_{n}\right\}_{n \geq 1}$ of open neighborhoods of $x_{0}$. It is still a fundamental system for $x_{0}$, as $U_{n} \subset V_{n}$ for all $n \geq 1$.

3. Algebraic genericity. We start by isolating two properties that will be often used. These properties relate to a measure space $(X, \mathcal{M}, \mu)$ :

$(\alpha) \inf \{\mu(A): A \in \mathcal{M}, \mu(A)>0\}=0$.

$(\beta) \sup \{\mu(A): A \in \mathcal{M}, \mu(A)<\infty\}=\infty$.

The exact conditions under which inclusions among the Lebesgue spaces $L^{p}(\mu, X)$ hold are well known. The assertions of the following theorem can be found in the paper [11] by J. L. Romero and in [10, Section 14.8].

Theorem 3.1. Let $(X, \mathcal{M}, \mu)$ be a measure space and $1 \leq p<q \leq \infty$.

(a) $L^{p}(\mu, X) \subset L^{q}(\mu, X)$ if and only if $\inf \{\mu(A): A \in \mathcal{M}, \mu(A)>0\}>0$.

(b) $L^{q}(\mu, X) \subset L^{p}(\mu, X)$ if and only if $\sup \{\mu(A): A \in \mathcal{M}, \mu(A)<\infty\}$ is finite.

For instance, if $\mu$ is a finite measure on $(X, \mathcal{M})$ and $\nu$ is the counting measure on an infinite set $Y$, then $L^{q}(\mu, X) \subset L^{p}(\mu, X)$ and $L^{p}(\mu, Y) \subset$ $L^{q}(\mu, Y)$ whenever $p<q$. In particular, we recover the inclusion relations $L^{q}(I) \subset L^{p}(I)(I=$ a bounded interval of $\mathbb{R})$ and $\ell_{p} \subset \ell_{q}$ as well as the non- 
inclusion relation $L^{r}(J) \not \subset L^{s}(J)(r, s \in[1, \infty]$ with $r \neq s, J$ an unbounded interval of $\mathbb{R}$ ).

REMARKS 3.2. 1. Note that the last theorem can be reformulated as follows:

- Let $1 \leq p<q \leq \infty$. Then $L^{p}(\mu, X) \backslash L^{q}(\mu, X) \neq \emptyset$ if and only if $(\alpha)$ holds.

- Let $1 \leq q<p \leq \infty$. Then $L^{p}(\mu, X) \backslash L^{q}(\mu, X) \neq \emptyset$ if and only if $(\beta)$ holds.

2. In [10, pp. 233-235] it is also proved that $(\alpha)$ is true if and only if there exists a sequence $\left(A_{n}\right)$ of pairwise disjoint measurable sets with $0<\mu\left(A_{n}\right)<1 / 2^{n}(n \in \mathbb{N})$, while $(\beta)$ holds if and only if there exists a sequence $\left(A_{n}\right)$ of pairwise disjoint measurable sets with $1<\mu\left(A_{n}\right)<\infty$ $(n \in \mathbb{N})$.

As a matter of fact, conditions $(\alpha)$ and $(\beta)$ will also turn out to be sharp conditions for much finer properties than the mere nonvacuousness of $L^{p} \backslash L^{q}$. This question will be studied in the present section, reaching more general conclusions than the ones by Aron et al. given in the Introduction. We first present the following concepts. Then we will state our main result.

Definition 3.3. Let $(X, \mathcal{M}, \mu)$ be a measure space and $p \in[1, \infty)$. The members of the set $L_{l \text {-strict }}^{p}:=L^{p}(\mu, X) \backslash \bigcup_{q \in[1, p)} L^{q}(\mu, X)$ will be called leftstrictly $p$-integrable functions. The members of the set $L_{r \text {-strict }}^{p}:=L^{p}(\mu, X) \backslash$ $\bigcup_{q \in(p, \infty]} L^{q}(\mu, X)$ will be called right-strictly p-integrable functions. Finally, the members of the set $L_{\text {strict }}^{p}:=L^{p}(\mu, X) \backslash \bigcup_{q \in[1, \infty] \backslash\{p\}} L^{q}(\mu, X)$ are said to be strictly $p$-integrable functions.

We notice that $L_{\text {strict }}^{p}=L_{l \text {-strict }}^{p} \cap L_{r \text {-strict }}^{p}, L_{l \text {-strict }}^{1}=L^{1}(\mu, X)$ and $L_{r \text {-strict }}^{1}=L_{\text {strict }}^{1}$.

Theorem 3.4. Assume that $p \in[1, \infty),(X, \mathcal{M}, \mu)$ is a measure space and $L^{p}(\mu, X)$ is separable.

(a) $L_{r \text {-strict }}^{p}$ is maximal dense-lineable if and only if $(\alpha)$ holds.

(b) If $p>1$, then $L_{l \text {-strict }}^{p}$ is maximal dense-lineable if and only if $(\beta)$ holds.

(c) If $p>1$, then $L_{\mathrm{strict}}^{p}$ is maximal dense-lineable if and only if both $(\alpha)$ and $(\beta)$ hold.

Proof. From Theorem 3.1 and Remark 3.2.1, it follows that conditions $(\alpha),(\beta)$ and $(\alpha) \&(\beta)$ are respectively necessary in (a), (b) and (c). We have to prove that they are also sufficient.

Suppose that $(\alpha)$ holds. First, we are going to demonstrate that $L_{r \text {-strict }}^{p}$ is $c$-lineable. According to Remark 3.2.2, we can select a sequence $\left(A_{n}\right) \subset \mathcal{M}$ 
of pairwise disjoint sets with $0<\mu\left(A_{n}\right)<1 / 2^{n}(n \geq 1)$. For each $a \in(1, \infty)$, consider the function $f_{a}: X \rightarrow[0, \infty)$ given by

$$
f_{a}=\sum_{n=1}^{\infty} \frac{1}{n^{1 / p}(\log (n+1))^{a / p} \mu\left(A_{n}\right)^{1 / p}} \cdot \chi_{A_{n}} .
$$

It is clear that $f_{a}$ is measurable. From the disjointness of the sets $A_{n}$, it follows that $f_{a}^{p}=\sum_{n=1}^{\infty} \frac{1}{n(\log (n+1))^{a} \mu\left(A_{n}\right)} \cdot \chi_{A_{n}}$. Moreover,

$$
\left\|f_{a}\right\|_{p}=\left[\int_{X} f_{a}^{p} d \mu\right]^{1 / p}=\left[\sum_{n=1}^{\infty} \frac{1}{n(\log (n+1))^{a}}\right]^{1 / p}=: \delta .
$$

Now, $\delta$ is finite: use, for instance, Cauchy's condensation principle. Thus $f_{a} \in L^{p}(\mu, X)$. Define

$$
M:=\operatorname{span}\left(\left\{f_{a}: a \in(1, \infty)\right\}\right) \subset L^{p}(\mu, X) .
$$

The functions $f_{a}$ are linearly independent. Indeed, suppose $c_{1} f_{\alpha_{1}}+\cdots+$ $c_{N} f_{\alpha_{N}}=0$, the $\alpha_{j}$ are pairwise distinct and not all the scalars $c_{j}$ are null. We can assume that $N \geq 2, \alpha_{1}<\cdots<\alpha_{N}$ and $c_{1} \neq 0$. Since $(\log (n+1))^{\alpha_{2}-\alpha_{1}}$ $\rightarrow \infty$ as $n \rightarrow \infty$, we can choose $n_{0} \geq 2$ such that

$$
(\log (n+1))^{\left(\alpha_{2}-\alpha_{1}\right) / p}>2 \sum_{j=2}^{N}\left|c_{j} / c_{1}\right| \quad\left(n \geq n_{0}\right) .
$$

In particular,

$$
\frac{\left|c_{1}\right|}{n_{0}^{1 / p}\left(\log \left(n_{0}+1\right)\right)^{\alpha_{1} / p} \mu\left(A_{n_{0}}\right)^{1 / p}}>\sum_{j=2}^{N} \frac{\left|c_{j}\right|}{n_{0}^{1 / p}\left(\log \left(n_{0}+1\right)\right)^{\alpha_{j} / p} \mu\left(A_{n_{0}}\right)^{1 / p}} .
$$

Therefore $\left|c_{1} f_{\alpha_{1}}\right|>\left|-\left(c_{2} f_{\alpha_{2}}+\cdots+c_{N} f_{\alpha_{N}}\right)\right|$ on $A_{n_{0}}$, which is absurd.

Furthermore, each $f \in M \backslash\{0\}$ happens not to belong to $L^{q}(\mu, X)$, for any $q>p$. Indeed, $f$ has the form $c_{1} f_{\alpha_{1}}+\cdots+c_{N} f_{\alpha_{N}}$ as in the last paragraph. Select an $n_{0} \in \mathbb{N}$ such that (6) holds. Let $n \geq n_{0}$. Then on $A_{n}$ we have

$$
\begin{aligned}
|f| & \geq\left|c_{1} f_{\alpha_{1}}\right|-\left(\left|c_{2} f_{\alpha_{2}}\right|+\cdots+\left|c_{N} f_{\alpha_{N}}\right|\right) \\
& =\frac{\left|c_{1}\right|}{n^{1 / p}(\log (n+1))^{\alpha_{1} / p} \mu\left(A_{n}\right)^{1 / p}}-\sum_{j=2}^{N} \frac{\left|c_{j}\right|}{n^{1 / p}(\log (n+1))^{\alpha_{j} / p} \mu\left(A_{n}\right)^{1 / p}} \\
& \geq \frac{\left|c_{1}\right|}{n^{1 / p}(\log (n+1))^{\alpha_{1} / p} \mu\left(A_{n}\right)^{1 / p}}-\frac{\sum_{j=2}^{N}\left|c_{j}\right|}{n^{1 / p}(\log (n+1))^{\alpha_{2} / p} \mu\left(A_{n}\right)^{1 / p}} \\
& \geq \frac{1}{2} \cdot \frac{\left|c_{1}\right|}{n^{1 / p}(\log (n+1))^{\alpha_{1} / p} \mu\left(A_{n}\right)^{1 / p}}
\end{aligned}
$$


Now, fix $q>p$. Then it follows that

$$
\begin{aligned}
\|f\|_{q}^{q} & \geq \sum_{n=n_{0}}^{\infty} \frac{1}{2^{q}} \cdot \frac{\left|c_{1}\right|^{q}}{n^{q / p}(\log (n+1))^{\alpha_{1} q / p} \mu\left(A_{n}\right)^{q / p}} \mu\left(A_{n}\right) \\
& \geq \sum_{n=n_{0}}^{\infty} \frac{1}{2^{q}} \cdot \frac{\left(2^{q / p-1}\right)^{n}\left|c_{1}\right|^{q}}{n^{q / p}(\log (n+1))^{\alpha_{1} q / p}}=\infty
\end{aligned}
$$

because the general term of this series is unbounded since $2^{q / p-1}>1$. Consequently, $f \notin L^{q}(\mu, X)$ whenever $q \in(p, \infty)$. The facts that $\mu\left(A_{n}\right)>0$ and

$$
d_{n}:=\frac{1}{2} \cdot \frac{\left|c_{1}\right|}{n^{1 / p}(\log (n+1))^{\alpha_{1} / p} \mu\left(A_{n}\right)^{1 / p}} \rightarrow \infty \quad \text { as } n \rightarrow \infty
$$

(because $\left.\mu\left(A_{n}\right)<1 / 2^{n}\right)$, together with the inequality $|f| \geq d_{n}$ on $A_{n}$ $\left(n \geq n_{0}\right)$, show that $f \notin L^{\infty}(\mu, X)$. Hence $M \backslash\{0\} \subset L_{r \text {-strict }}^{p}$. So far, we have proved that the last set is $c$-lineable. To see that it is in fact maximal dense-lineable, it is enough to observe that $L_{r \text {-strict }}^{p}=\bigcap_{S \in \Gamma}(E \backslash S)$, where $E=L^{p}(\mu, X)$ and $\Gamma$ is the family of all subspaces $L^{p}(\mu, X) \cap L^{q}(\mu, X)$ $(q>p)$. Note that $\bigcap_{S \in \Gamma} S$ is dense in $E$ because it contains the class $S_{t}$ of step functions. By applying Lemma 2.1, the proof of (a) is concluded.

Part (b) is proved in a similar way, only by taking into account that this time the sets $A_{n}$ can be chosen such that $\mu\left(A_{n}\right)>1$ for all $n$ (see Remark 3.2 ). Then the above subspace $M$ works, and a further use of Lemma 2.1 provides the desired maximal dense-lineability. The unique difference is the business of proving that $f \notin L^{q}(\mu, X)$ whenever $q<p$, where $f$ is as before. This is easy, because we now have

$$
\begin{aligned}
\|f\|_{q}^{q} & \geq \sum_{n=n_{0}}^{\infty} \frac{1}{2^{q}} \cdot \frac{\left|c_{1}\right|^{q}}{n^{q / p}(\log (n+1))^{\alpha_{1} q / p} \mu\left(A_{n}\right)^{q / p}} \cdot \mu\left(A_{n}\right) \\
& \geq \sum_{n=n_{0}}^{\infty} \frac{1}{2^{q}} \cdot \frac{\left|c_{1}\right|^{q}}{n^{q / p}(\log (n+1))^{\alpha_{1} q / p}} .
\end{aligned}
$$

This series diverges, for $q / p<1$. Therefore $\|f\|_{q}=\infty$, as required.

It remains to demonstrate the "if" part of (c). Again by Remark 3.2.2 and $(\alpha)$, there are infinitely many pairwise disjoint measurable sets $A_{n}$ with $0<\mu\left(A_{n}\right)<1 / 2^{n}(n \in \mathbb{N})$. Observe that the set $A:=\bigcup_{n=1}^{\infty} A_{n}$ has finite measure. It follows that $(\beta)$ is also satisfied by the measure subspace $\left(X \backslash A, \mathcal{M}_{X \backslash A},\left.\mu\right|_{X \backslash A}\right)$. This entails the existence of infinitely many mutually disjoint measurable sets $B_{n}$ with $1<\mu\left(B_{n}\right)<\infty$ and $A_{k} \cap B_{n}=\emptyset$ $(n, k \in \mathbb{N})$. Let $C_{1}:=A_{1}, C_{2}:=B_{1}, C_{3}:=A_{2}, C_{4}:=B_{2}, \ldots$ and

$$
\begin{aligned}
M & :=\operatorname{span}\left(\left\{f_{\alpha}: \alpha \in(1, \infty)\right\}\right), \quad \text { where } \\
f_{\alpha} & =\sum_{n=1}^{\infty} \frac{1}{n^{1 / p}(\log (n+1))^{\alpha / p} \mu\left(C_{n}\right)^{1 / p}} \cdot \chi_{C_{n}} .
\end{aligned}
$$


The proof is now an appropriate combination of the approaches of (a) and (b). The details (cumbersome, but easy) are left to the reader.

REMARKS 3.5. 1. The separability of $L^{p}(\mu, X)$ is a general hypothesis in the last theorem. According to Proposition 2.3, this condition is fulfilled if $(\sigma)$ holds.

2 . In the case $p=1$, trivially, $L_{l \text {-strict }}^{1}$ is always maximal dense-lineable, while (by (a)) $L_{\text {strict }}^{1}$ is maximal dense-lineable if and only if $(\alpha)$ holds.

3 . The last theorem is no longer valid if $p=\infty$. Indeed, neither $(\alpha)$ nor $(\beta)$ is compatible with the separability of $L^{\infty}(\mu, X)$ : see Proposition 2.4.

We conclude this section with the following corollary, which provides two examples of when the main theorem applies.

Corollary 3.6. Assume that $p \in[1, \infty),(X, \mathcal{M}, \mu)$ is a measure space and $L^{p}(\mu, X)$ is separable. Then $L_{r \text {-strict }}^{p}$ is maximal dense-lineable if one of the following properties is true:

(a) The measure $\mu$ is semifinite and nonatomic.

(b) $X$ is a $T_{1}$ topological space and there is a non-isolated point $x_{0} \in X$ such that $X$ is regular and first-countable at $x_{0}, \mu\left(\left\{x_{0}\right\}\right)=0$ and there is an open neighborhood $U$ of $x_{0}$ with $\mu(U)<\infty$ and $\mu(V)>0$ for any nonempty open set $V \subset U$.

Proof. If (a) is true, then $[0, \mu(M)]=\{\mu(A): A \in \mathcal{M}$ and $A \subset M\}$ for every set $M \in \mathcal{M}$ (see for instance [10, Theorem 11.27]). Hence $(\alpha)$ is satisfied and Theorem 3.4 applies. If we start from (b), it follows from Lemma 2.5 that there exists a fundamental system $\left\{U_{n}\right\}_{n \geq 1}$ of open neighborhoods of $x_{0}$ such that $\overline{U_{n+1}} \subset U_{n} \subset U$ and $U_{n} \backslash \overline{U_{n+1}} \neq \emptyset$ for all $n \in \mathbb{N}$. Therefore the "annuli" $A_{n}:=U_{n} \backslash \overline{U_{n+1}}$ satisfy $\mu\left(A_{n}\right)>0(n \geq 1)$. Since $\left(U_{n}\right)$ is a decreasing sequence with intersection $\left\{x_{0}\right\}$ (because $X$ is $T_{1}$ ) and the $U_{n}$ 's have finite measure, one derives that $\lim _{n \rightarrow \infty} \mu\left(U_{n}\right)=\mu\left(\left\{x_{0}\right\}\right)=0$ (see [12, Chap. 1]). Hence $\mu\left(A_{n}\right) \rightarrow 0$, which shows that $(\alpha)$ is again fulfilled.

According to Proposition 2.3(b), the separability of $L^{p}(\mu, X)$ is guaranteed by a set of conditions, some of which are finer than those given in part (b) of the last corollary.

4. Nonintegrability on any open set. This short section is devoted to showing that under appropriate, rather mild, conditions on a regular measure, right-strictness can be reinforced to involve every nonempty open set.

THEOREM 4.1. Let $X$ be a Hausdorff first-countable separable locally compact perfect topological space. Assume that $\mu$ is a Borel measure on $X$ 
that is continuous, regular and has full support. Let $p \in[1, \infty)$ and $\mathcal{C}$ be the set of all $f \in L^{p}(\mu, X)$ such that, for every nonempty open subset $U$ of $X$ and every $q>p, f \notin L^{q}(\mu, U)$. Then $\mathcal{C}$ is a dense subset of $L^{p}(\mu, X)$.

Proof. Consider the space $E:=L^{p}(\mu, X)$ as a topological group under the operation + . If $\tau$ is the topology of $X$, our set $\mathcal{C}$ can be written as $\mathcal{C}=\bigcap_{S \in \Gamma}(E \backslash S)$, where $\Gamma:=\left\{\left\{f \in L^{p}(\mu, X):\left.f\right|_{U} \in L^{q}(\mu, U)\right\}: q>p\right.$ and $U \in \tau \backslash\{\emptyset\}\}$. Observe that each member of $\Gamma$ is a subgroup of $E$. Moreover, the set $\bigcap_{S \in \Gamma} S$ is dense in $E$, for it contains the set $S_{t}$ of step functions. According to Lemma 2.2, it is enough to show that $\mathcal{C} \neq \emptyset$.

To this end, observe that our hypotheses on $X$ and $\mu$ imply that the conditions in part (b) of Corollary 3.6 are satisfied at all points $x_{0} \in X$. As in the proof of that corollary, we can consider an appropriate decreasing countable basis $U_{n}=U_{n, x_{0}}(n \geq 1)$ of open neighborhoods for $x_{0}$, and then select mutually disjoint measurable subsets $A_{n}=A_{n, x_{0}}(n \in \mathbb{N})$ of positive measure such that $\mu\left(A_{n, x_{0}}\right) \rightarrow 0$. By passing to a subsequence if necessary, one may suppose that $0<\mu\left(A_{n, x_{0}}\right)<1 / 2^{n}$ for every $n$. Similarly to the proof of Theorem 3.4, we consider the measurable function $f_{x_{0}}: X \rightarrow[0, \infty)$ given by

$$
f_{x_{0}}=\sum_{n=1}^{\infty} \frac{1}{n^{1 / p}(\log (n+1))^{2 / p} \mu\left(A_{n, x_{0}}\right)^{1 / p}} \cdot \chi_{A_{n, x_{0}}} .
$$

As in that theorem (with $\alpha=2$ ), we get

$$
\left\|f_{x_{0}}\right\|_{p}=\left[\sum_{n=1}^{\infty} \frac{1}{n(\log (n+1))^{2}}\right]^{1 / p}=: \beta<\infty .
$$

Observe that $\beta$ does not depend on $x_{0}$. At this point, the separability of $X$ comes into play, providing a dense countable subset $\left\{x_{k}: k \geq 1\right\} \subset X$. Define the measurable function $f: X \rightarrow[0, \infty)$ by

$$
f=\sum_{k=1}^{\infty} 2^{-k} f_{x_{k}}=\sum_{k=1}^{\infty} \sum_{n=1}^{\infty} \frac{2^{-k}}{n^{1 / p}(\log (n+1))^{2 / p} \mu\left(A_{n, x_{k}}\right)^{1 / p}} \cdot \chi_{A_{n, x_{k}}} .
$$

Our final task is to show that $f \in \mathcal{C}$. From the Minkowski inequality,

$$
\|f\|_{p} \leq \sum_{k=1}^{\infty}\left\|2^{-k} f_{x_{k}}\right\|_{p}=\sum_{k=1}^{\infty} 2^{-k} \beta=\beta<\infty .
$$

Hence $f \in L^{p}(\mu, X)$.

Our proof will be concluded as soon as we show that, for every $q>p$ and every $U \in \tau \backslash\{\emptyset\}, f \notin L^{q}(\mu, U)$. For this, fix such $q$ and $U$. From the density of $\left(x_{k}\right)$ it follows that there is $k_{0} \in \mathbb{N}$ with $y:=x_{k_{0}} \in U$. Since the $U_{n, y}$ 's form a decreasing basis of open neighborhoods of $y$, we can find an 
$n_{0} \in \mathbb{N}$ such that $U_{n, y} \subset U$ for all $n \geq n_{0}$. Hence $A_{n, y} \subset U$ for such $n$. Since all terms in (7) are nonnegative, we derive

$$
|f|^{q} \geq \sum_{n=n_{0}}^{\infty} \frac{2^{-k_{0} q}}{n^{q / p}(\log (n+1))^{2 q / p} \mu\left(A_{n, y}\right)^{q / p}} \cdot \chi_{A_{n, y}},
$$

from which one deduces that

$$
\begin{aligned}
\|f\|_{q}^{q} & \geq 2^{-k_{0} q} \cdot \sum_{n=n_{0}}^{\infty} \frac{1}{n^{q / p}(\log (n+1))^{2 q / p} \mu\left(A_{n, y}\right)^{q / p-1}} \\
& \geq 2^{-k_{0} q} \cdot \sum_{n=n_{0}}^{\infty} \frac{\left(2^{q / p-1}\right)^{n}}{n^{q / p}(\log (n+1))^{2 q / p}}=\infty,
\end{aligned}
$$

because $q / p-1>0$. This proves the desired conclusion if $q$ is finite. If $q=\infty$, suffice it to observe that $\inf _{A_{n, y}}|f| \rightarrow \infty(n \rightarrow \infty)$ and that $\mu\left(A_{n, y}\right)>0$ for all $n \in \mathbb{N}$.

Observe that, from the proof of Lemma 2.2, we obtain a dense affine linear subspace of functions with the property described in the last theorem. On the other hand, as in Corollary 3.6, we cannot expect left-strictness in the conclusion of Theorem 4.1 because our measure may well be finite.

5. Final remarks. 1. The nice notion of " $A$ stronger than $B$ " introduced in [2] can also be used when dealing with the problem of denselineability.

2. If the separability of $L^{p}(\mu, X)$ is not guaranteed, we at least keep $c$ lineability in the conclusions of Theorem 3.4 and Corollary 3.6. By trivially extending the strictness to the case $p=\infty$, we also have $c$-lineability here. We pose it as an open problem to study the dense-lineability and maximal dense-lineability in this case.

3. We also want to ask whether the special sets studied in this paper are residual/spaceable under appropriate conditions. Moreover, we do not know whether the set $\mathcal{C}$ considered in Theorem 4.1 is lineable/c-lineable/denselineable/maximal-dense lineable.

4. Regarding that theorem, it is worth warning the reader that the expression " $f \notin L^{q}(\mu, U)$ for every nonempty open set $U$ " cannot be replaced by " $f \notin L^{q}(\mu, A)$ for every measurable set $A$ with $\mu(A)>0$ ". Indeed, since $f$ is in $L^{p}(\mu, X)$, it is finite $\mu$-almost everywhere. Thus there is a set $Z$ with $\mu(Z)=0$ such that $X=Z \cup \bigcup_{n=1}^{\infty}\{x \in X:|f(x)| \leq n\}$. Hence some set $\left\{x \in X:|f(x)| \leq n_{0}\right\}=: A$ has positive measure. But $|f|^{q} \leq n_{0}^{q-p}|f|^{p}$ on $A$, so $f \in L^{q}(\mu, A)$. 
5. In [4 the following concept is introduced. A subset $A$ of a topological vector space of scalar functions is called algebrable if $A \cup\{0\}$ contains an infinitely generated algebra. We cannot expect algebrability in our setting. For instance, if $q>p \geq 1$ and $\mu$ is finite, then $A:=L^{p}(\mu, X) \backslash L^{q}(\mu, X)$ is not algebrable. Indeed, if it were, there would exist $f \in A$ with $f^{N} \in A$ for all $N \in \mathbb{N}$. Choose $N$ with $N p>q$. Then $f \in L^{N p}(\mu, X) \subset L^{q}(\mu, X)$, which is absurd.

6. Lineability properties of families of functions that are either Riemann integrable or non-Riemann integrable are studied in [7].

Acknowledgements. The author is indebted to the referee for helpful comments and suggestions.

This research was partly supported by the Plan Andaluz de Investigación de la Junta de Andalucía FQM-127, by MCI Grant MTM2009-10696-C02-01 and by MCYT (Acción Especial) MTM2007-30904-E.

\section{References}

[1] R. Aron, D. García and M. Maestre, Linearity in non-linear problems, Rev. R. Acad. Cien. Ser. A Mat. 95 (2001), 7-12.

[2] R. Aron, F. J. García-Pacheco, D. Pérez-García and J. B. Seoane-Sepúlveda, On dense-lineability of sets of functions on $\mathbb{R}$, Topology 48 (2009), 149-156.

[3] R. M. Aron, V. I. Gurariy and J. B. Seoane-Sepúlveda, Lineability and spaceability of sets of functions on $\mathbb{R}$, Proc. Amer. Math. Soc. 133 (2005), 795-803.

[4] R. M. Aron, D. Pérez-García and J. B. Seoane-Sepúlveda, Algebrability of the set of non-convergent Fourier series, Studia Math. 175 (2006), 83-90.

[5] F. Bayart, Topological and algebraic genericity of divergence and universality, ibid. 167 (2005), 161-181.

[6] L. Bernal-González, Dense-lineability in spaces of continuous functions, Proc. Amer. Math. Soc. 136 (2008), 3163-3169.

[7] F. J. García-Pacheco, M. Martín and J. B. Seoane-Sepúlveda, Lineability, spaceability, and algebrability of certain subsets of function spaces, Taiwanese J. Math. 13 (2009), 1257-1269.

[8] V. Gurariy and L. Quarta, On lineability of sets of continuous functions, J. Math. Anal. Appl. 294 (2004), 62-72.

[9] G. A. Muñoz-Fernández, N. Palmberg, D. Puglisi and J. B. Seoane-Sepúlveda, Lineability in subsets of measure and function spaces, Linear Algebra Appl. 428 (2008), 2805-2812.

[10] O. A. Nielsen, An Introduction to Integration and Measure Theory, Wiley, New York, 1997.

[11] J. L. Romero, When is $L^{p}(\mu)$ contained in $L^{q}(\mu)$ ?, Amer. Math. Monthly 90 (1983), 203-206. 
[12] W. Rudin, Real and Complex Analysis, 3rd ed., McGraw-Hill, New York, 1987.

Luis Bernal-González

Facultad de Matemáticas

Universidad de Sevilla

41080 Sevilla, Spain

E-mail: lbernal@us.es

Received January 21, 2010

Revised version May 5, 2010 